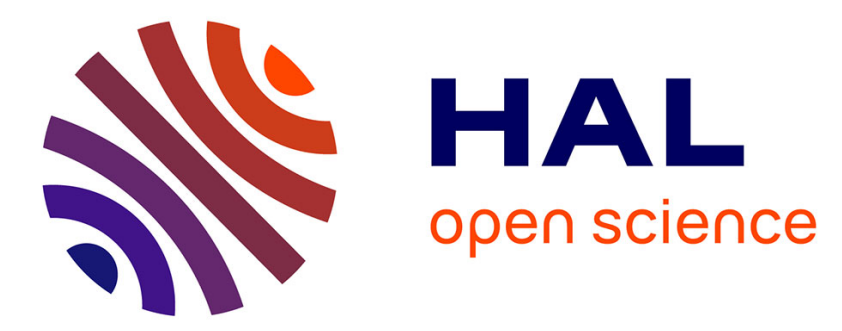

\title{
Circumferential guided wave measurements of a cylindrical fluid-filled bone-mimicking phantom
}

Pierre Nauleau, Quentin Grimal, Jean-Gabriel Minonzio, Pascal Laugier, Claire Prada

\section{- To cite this version:}

Pierre Nauleau, Quentin Grimal, Jean-Gabriel Minonzio, Pascal Laugier, Claire Prada. Circumferential guided wave measurements of a cylindrical fluid-filled bone-mimicking phantom. Journal of the Acoustical Society of America, 2014, 135 (2), pp.994 10.1121/1.4861366 . hal-01301185

\section{HAL Id: hal-01301185 https://hal.sorbonne-universite.fr/hal-01301185}

Submitted on 11 Apr 2016

HAL is a multi-disciplinary open access archive for the deposit and dissemination of scientific research documents, whether they are published or not. The documents may come from teaching and research institutions in France or abroad, or from public or private research centers.
L'archive ouverte pluridisciplinaire HAL, est destinée au dépôt et à la diffusion de documents scientifiques de niveau recherche, publiés ou non, émanant des établissements d'enseignement et de recherche français ou étrangers, des laboratoires publics ou privés. 


\title{
Circumferential guided wave measurements of a cylindrical fluid-filled bone-mimicking phantom
}

\author{
Pierre Nauleau, Quentin Grimal ${ }^{1}$, Jean-Gabriel Minonzio, and Pascal Laugier \\ UPMC Univ Paris 06, CNRS 7623 \\ Laboratoire d'Imagerie Paramtrique \\ 15 rue de l'cole de mdecine, F-75006 Paris, France
}

Claire Prada

Institut Langevin, ESPCI Paris Tech

Universit Denis Diderot Paris 7, CNRS 7587

1 rue Jussieu, F-75005 Paris, France

\footnotetext{
${ }^{1}$ e-mail: quentin.grimal@upcm.fr
} 


\begin{abstract}
In the context of hip fracture risk prediction, measurement of guided waves could improve the assessment of cortical femoral neck properties. The decomposition of the time reversal operator (DORT) method was previously shown to be efficient to measure circumferential guided modes in an empty cortical bone-mimicking tube of circular cross-section. In this study, an adaptation of the DORT method is proposed to probe the same bone-mimicking tube but filled with a marrow-mimicking fluid. The contributions to the backscattered field of waves multiply reflected in the cavity of the tube interfere with those of circumferential guided waves. The former contributions are eliminated in the backpropagation image using ad-hoc criterion determined with simulation. Eight portions of different guided modes were observed from experimental and simulated data. They were identified by comparison with theoretical predictions. This work confirms the feasibility of measuring guided waves in a fluid-filled tube of bone-mimicking material with the DORT method.
\end{abstract}




\section{Introduction}

Osteoporosis is a systemic disease characterized by a compromised bone strength implying an increased risk of fracture. The economic and human impact of osteoporosis-related fractures is significant: in the five largest countries of Europe, $21 \%$ of all women aged $50-84$ years are estimated to have osteoporosis and the economic burden is approximated at 30.7

billion $€ .^{1}$ The standard method for diagnosis of osteoporosis and prediction of fracture risk consists in assessing bone mineral density (BMD) using ionizing dual X-ray absorptiometry (DXA). However, growing evidence indicates that low BMD is not the sole factor accounting for fracture risk. ${ }^{2,3}$ The propagation characteristics of ultrasonic waves in bone being closely related to its structure and to its elastic properties, quantitative ultrasound (QUS) techniques have been developped in the past two decades to overcome limitations of DXA. ${ }^{4}$ Hip fractures are associated with the highest morbidity and mortality. ${ }^{1}$ The risk of hip fracture is best predicted with DXA measurements performed directly at the femoral neck. ${ }^{5}$ Similarly, we hypothesize that QUS evaluation at the proximal femur could achieve a more accurate risk prediction than peripheral QUS measurements. Recent reports have evidenced the feasibility and the relevance of in vivo QUS measurements at this specific site. ${ }^{6}$ However, although the contribution of cortical bone to the strength of the femoral neck has been evidenced in recent studies, ${ }^{7,8}$ the specific assessment of cortical bone at the hip remains highly challenging with currently available DXA or QUS technologies. 
Following the recent developments of the axial transmission technique that exploits the waveguide character of the cortical envelope of long bones, ${ }^{9}$ our group has proposed to extend the guided wave approach to the cortical shell of the femoral neck. A numerical study has revealed that cortical bone of the neck behaves as a waveguide supporting the propagation of circumferential guided waves. ${ }^{10}$ In an ex vivo experimental study, the time-of-flight of the first arriving circumferential ultrasonic wave transmitted through human femoral neck specimens was found to be correlated to mechanical strength. ${ }^{11}$

In the appropriate frequency range (200 kHz-1.5 MHz approximately), the cortical bone shell is a multi-modal waveguide, which means that different modes coexist in the same waveguide. ${ }^{12}$ The velocity of each guided mode reflects a specific combination of several bone parameters, such as bone thickness and elasticity. We hypothesized that the identification of the different modes and the measurement of their frequency-dependent velocity combined with an appropriate propagation model should allow estimating cortical bone thickness and anisotropic elastic properties, which are key factors to predict bone mechanical strength. Thus, in contrast with our previous studies ${ }^{10,11}$ that were limited to the measurement of the first arriving signal, we aim at measuring the full response of the waveguide.

Towards this goal, we demonstrated the feasibility of measuring the circumferential dispersion curves of an empty cylindrical bone-mimicking phantom ${ }^{13}$ in a non-contact configuration using the DORT method (French acronym standing for Decomposition of the Time Reversal 
Operator) based on the diagonalization of the time reversal operator. ${ }^{14,15}$

The objective of the present study is to adapt the DORT method in the case where the cavity delimited by the cortical shell is filled with a marrow-mimicking fluid. In this case, the circumferential guided wave signals radiated towards the array interfere with waves transmitted through the fluid-filled cavity and multiply reflected on the cavity walls. A method is proposed to filter out the contributions of the latter reflections. This adaptation of the DORT method is used to retrieve portions of the dispersion curves from experimentally recorded backscattered signals from a cylindrical bone-mimicking phantom filled with a marrow-mimicking fluid. Experimental portions of dispersion curves are compared with portions obtained from simulated backscattered signals and with theoretical dispersion curves.

\section{Rationale for dispersion curve measurements with the DORT method}

The principle of the method, extensively described elsewhere ${ }^{15,16}$ is summarized here.

\section{A. Scattering of acoustic waves by a cylinder}

A transmitter-receiver array composed of $N$ transducers is placed in a water tank to measure a tube of diameter $D$. The median axis, parallel to the array, is labelled $\Delta$ in the following. This object is insonified with a harmonic plane wave emitted by the array, in the $\mathrm{x}$ direction (Fig. 1). At a specific incidence angle $\theta$ corresponding 
to two symmetrical points $A$ and $B$, two circumferential guided waves of phase velocity $c_{\varphi}(f)$ are generated. The incidence angle $\theta$ is determined by the SnellDescartes law:

$$
\sin \theta(f)=\frac{c_{0}}{c_{\varphi}(f)},
$$

with $c_{0}$, the sound velocity in water. The two generated waves propagate being guided by the shell, the wave generated in $A$ propagating clockwise and the wave generated in $B$ propagating counterclockwise. During the propagation in the tube, these circumferential waves radiate into the external medium with the angle $\theta$. The radiated waves received by the array come from $B$ for the clockwise propagation and $A$ for the counterclockwise propagation. The propagation path of the circumferential wave generated in $A$ is depicted in Fig. 1, the path of the wave generated in $B$ being symmetrical. This observation is only true in the far field. In fact, the circumferential waves received by the array not only radiate from a point $A$ or $B$, but from a small area around these points. It can be shown using geometrical approximation that the radiated waves come from two apparent radiation points: two virtual sources $A^{\prime}$ and $B^{\prime}$. These points are located on the line $\Delta$ and aligned on $\mathrm{x}$ with $A$ and $B .{ }^{17}$

The distance between $A^{\prime}$ and $B^{\prime}$ can be related to the diameter of the tube and the angle $\theta$ using trigonometry: $d_{A^{\prime} B^{\prime}}=D \sin \theta$. The phase velocity can thus be 
related to the distance between the two virtual sources by

$$
c_{\varphi}(f)=c_{0} \frac{D}{d_{A^{\prime} B^{\prime}}(f)} .
$$

The phase velocity being dependent of the frequency, the position of $A^{\prime}$ and $B^{\prime}$ evolves with the frequency.

Hence, the key point of this guided wave measurement is to locate the virtual sources, the apparent radiation points $A^{\prime}$ and $B^{\prime}$ for several modes, at several frequencies.

\section{B. The DORT method}

The DORT method applied to backscatter measurements of circumferential guided waves with a N-element array can be summed up in four steps. (i) The interelement array response matrix is measured: signals scattered by the tube for $N$ successive independent emissions are recorded by the $N$ elements of the array. Time-domain Fourier transform is applied to each signal to obtain the three-dimensional array transfer matrix of the system of dimensions $N \times N \times N_{f}$, with $N_{f}$ the number of frequency samples. (ii) A singular value decomposition is applied to the two-dimensional transfer matrix, of dimensions $N \times N$, at each frequency. (iii) Singular vectors are selected based on considerations described below. (iv) These vectors are backpropagated at each 
frequency. Step (iv) which is performed numerically in our experiment, is equivalent to the time-reversal process. It consists in calculating the backpropagated wavefield, in an area $(x, y)$ containing the object, resulting from the emission of singular vectors of the time-reversal operator. (v) The distance between virtual sources is measured on this backpropagation image and is converted into phase velocity (or wavenumber) using Eq.(2).

More precisely, the backpropagated wavefield is observed on the line $\Delta$ which corresponds to the theoretical location of apparent radiation points $A^{\prime}$ and $B^{\prime}$ (Fig. 1). The wavefield amplitude along this line, collected at each frequency $(f)$ is then displayed in the plane $(y, f)$. Examples of backpropagation images in (x,y)-plane, at 0.6 and $1 \mathrm{MHz}$ and in (f,y)-plane are presented (Fig. 2). They have been obtained from the simulation of the backscatter experiment for a steel tube. This example was chosen because the phenomena are more clearly illustrated than in a bone-mimicking material, due to the higher amplitudes of the backscattered signals.

The criterion for the selection of the singular vectors follows from the analysis of the invariants of the time-reversal operator. Prada et al. have shown that the process of generation and reception of the circumferential guided waves in the backscatter experiment is invariant through the time-reversal process. ${ }^{14,15}$ For- 
mally, these invariants correspond to the singular vectors of the array transfer matrix. Thus, a guided mode, i.e. two circumferential guided waves propagating clockwise and counterclockwise, is associated with a pair of singular vectors. For example, if three modes are expected for the studied waveguide, six singular vectors will be selected at the step (iii).

\section{Material and methods}

\section{A. Bone phantom}

The model considered in this study consists of a cortical bone-mimicking tube filled with a marrow-mimicking fluid. The cortical part of the phantom is a hollow cylinder made of a commercial cortical bone-mimicking material (Sawbones, Pacific Research Laboratory Inc., Vashon, WA). This material consists of short glass fibers embedded in an epoxy resin. The fibers of the manufactured tube are aligned with the axis of the cylinder. Thus, this composite presents a transverse isotropy, the plane of symmetry being perpendicular to the tube axis. In this study, we are interested in circumferential waves propagating in the plane of isotropy. Material properties of this mock bone were previously measured ${ }^{9}$ and found to be representative of the mean properties generally reported for human cortical bone ${ }^{18,19}$ (Table 1). The diameter and the wall thickness of the tube are $26 \mathrm{~mm}$ and $2.1 \mathrm{~mm}$, which 
Table 1: Comparison of longitudinal, transverse velocities, mass densities ${ }^{9,18}$ and attenuations $^{9,19}$ of cortical bone-mimicking material and human cortical bone (mid-diaphysis of the femur), in the plane of isotropy. $L$ and $T$ stand for longitudinal and transverse components.

$\begin{array}{ccc} & \text { Mock bone } & \text { Human bone } \\ c_{L}\left(\mathrm{~m} . \mathrm{s}^{-1}\right) & 2870 & 3205 \\ c_{T}\left(\mathrm{~m} . \mathrm{s}^{-1}\right) & 1520 & 1495 \\ \rho\left(\mathrm{kg} \cdot \mathrm{m}^{-3}\right) & 1640 & 1879 \\ \alpha_{L}\left(\mathrm{~dB} . \mathrm{cm}^{-1} \cdot \mathrm{MHz}^{-1}\right) & 2.63 & 2.56 \\ \alpha_{T}\left(\mathrm{~dB} . \mathrm{cm}^{-1} \cdot \mathrm{MHz}^{-1}\right) & 8.40 & \text { Unknown }\end{array}$

are relatively close to standard geometrical properties of femoral neck. ${ }^{20}$

The presence of bone marrow was simulated by adding a fluid in the tube. There seems to be no consensus in literature on a marrow-mimicking fluid. We decided to use glycerol whose properties (velocity of $1900 \mathrm{~m} \cdot \mathrm{s}^{-1}$ and density of $1260 \mathrm{~kg} \cdot \mathrm{m}^{-3}$ ) results in a slightly higher acoustical impedance compared to that of marrow ${ }^{21}$ (2.39 vs. 1.39 $\left.\mathrm{MPa} \cdot \mathrm{m} \cdot \mathrm{s}^{-1}\right)$. However the attenuation coefficient is similar $\left(0.2 \mathrm{vs} .0 .15 \mathrm{~dB} \cdot \mathrm{cm}^{-1}\right.$ at $1 \mathrm{MHz})$.

B. Experimental acquisition 
The measurement of the transfer matrix was performed with a linear array of 128 cylindrically focused elements (Imasonic, Besançon, France). Its main characteristics are a focal length of $160 \mathrm{~mm}$, an array pitch of $1.1 \mathrm{~mm}$, a $-6 \mathrm{~dB}$ beam width of $4 \mathrm{~mm}$, a center frequency of $1 \mathrm{MHz}$ and a $-6 \mathrm{~dB}$ bandwidth of $0.6 \mathrm{MHz}$. The probe was used in a reflection mode: 128 excitations are performed successively, each followed by the recording of scattered signals by each of the 128 array elements. The center of the phantom is placed at the focal length of the probe, i.e. $160 \mathrm{~mm}$. The water tank containing the probe and the phantom was large enough (volume $1.40 \mathrm{~m} \times 1 \mathrm{~m} \times 0.50 \mathrm{~m}$ ), so that reflections on the tank walls do not disturb the measurements of circumferential waves.

The excitation signals have been chosen carefully to overcome the strong attenuation of the cortical bone-mimicking material. A classical emission of short pulses in a canonical basis (each element takes turns to emit a pulse) does not excite the phantom with a sufficient energy to record radiated contributions of circumferential waves. In order to increase the signal-to-noise ratio, Hadamard-Walsh basis was preferred to the canonical basis. In this basis, all elements emit simultaneously for each of the 128 excitations: some elements emit the excitation signal and the other ones emit its opposite according to the Hadamard-Walsh matrix (composed of 1 and -1 ). The excitation signal was a chirp, i.e. a pulse compression by linear frequency modulation was used to emit signals with sufficient energy without degrading resolution. The emitted chirp swept a 
frequency range of $[0.4-1.6] \mathrm{MHz}$ in $20 \mu \mathrm{s}$. A multi-channel electronic device (Lecoeur Electronique, Chuelles, France) was used to manage the 128 elements of the probe.

The signals reflected after each excitation were recorded during $100 \mu$ s by the 128 elements and digitized at a rate of $20 \mathrm{MHz}$. To increase the signal-to-noise ratio, the recorded signals were averaged 20 times. Finally, the interelement response matrix was Fourier transformed to obtain the transfer matrix of the system. Data were stored on a computer for off-line analysis.

\section{Simulation of the acquisition}

Numerical simulations are a useful tool to evaluate the potential of the method in ideal conditions. Simulations are also appropriate to gain a better insight into the experiment and to develop adapted signal processing, see section .

The simulation consists in analytical calculations of the signals scattered by the phantom and recorded by the array, in a $2 \mathrm{D}$ approximation. These signals are computed from the normal mode expansion method. ${ }^{22,23}$ The Fourier transform, $S_{i j}(f)$, of the signal received by the j-th element of the array after an excitation of the tube by the i-th element can be written as the sum of a certain number, $m+1$, of cylindrical normal modes of vibrations for a large elastic tube. The number $m$, is chosen to ensure the sum convergence. ${ }^{24}$ Each normal mode is weighted by a scattering coefficient, $R_{n}(f)$, which depends on the 
frequency, the elastic properties of the scatterer and the inner and surrounding fluids.

$$
S_{i j}(f)=\sum_{n=0}^{m} \epsilon_{n} R_{n}(f) H_{n}^{(1)}\left(k_{0} r_{i}\right) H_{n}^{(1)}\left(k_{0} r_{j}\right) \cos \left(n \alpha_{i j}\right),
$$

with $1<i, j<128$. The time-domain signals, $s_{i j}(t)$ are calculated as the inverse Fourier transform of $S_{i j}(f)$.

$$
s_{i j}(t)=\operatorname{Re}\left(\sum_{f=1}^{N_{f}} e^{-i 2 \pi f t} B(f) O_{i} O_{j} S_{i j}(f)\right) .
$$

In the normal modes expansion, the cylindrical Hankel functions of the first kind, $H_{n}^{(1)}$, depend on the wavenumber, $k_{0}$, in the surrounding fluid and on the distance between the emitter (respectively the receiver) and the tube, $r_{i}$ (resp. $r_{j}$ ). The n-th cylindrical normal mode depends on the angle $\alpha_{i j}$ formed by the lines between the tube and the emitter, on the one hand and the tube and the receiver, on the other hand. The Neumann coefficient $\epsilon_{n}$ verifies $\epsilon_{0}=1$ and $\epsilon_{n}=2$ for $n \geq 1$. The characteristics of the array, frequency bandwidth $B(f)$ and emission and reception aperture functions $O_{i}$ and $O_{j}$ are also taken into account. The simulated signals are combined according to the Hadamard-Walsh basis to obtain a transfer matrix of the system similar to the experimental one. The same signal processing (described in section ) is applied to simulated and experimental transfer matrices.

\section{Calculation of theoretical dispersion curves}


The software Disperse (Imperial College, London, UK) was used to calculate the theoretical dispersion curves. This software is based on the global matrix method, which involves the construction of a single matrix describing wave propagation and boundary conditions for each layer. The points of the dispersion curves are obtained by finding the values of the parameters $k$ and $f$ for which the determinant of the global matrix is zero. ${ }^{25}$ This software also provides attenuation, phase and group velocities and shapes of the modes at each frequency.

Disperse does not provide the dispersion curves for the particular waveguide investigated here: a tube loaded by two different fluids. As a proxy, we calculated with Disperse the dispersion curves of a plate loaded with infinite layers of water on one side and glycerol on the other side. Disperse was fed with the material properties of cortical bone-mimicking and marrow-mimicking materials mentionned in section . Loading the plate by glycerol and water significantly affects the dispersion curve appearance. When compared with the free plate modes, the dispersion curves of the loaded plate are slightly modified and two additional fluid-born modes, labelled $\mathrm{A}$ and $\mathrm{S}$, appear. Labelling of the different modes is performed by comparing, at each frequency, the deformation patterns of the free plate and of the immersed plate. The additional modes A and S, being non-attenuated, ${ }^{26}$ can be easily identified. Lamb modes are numbered according to their cut-off frequency.

\section{E. Signal processing}


In this experiment with a fluid-filled phantom, a major contribution to the received signals comes from multiple reflections on the walls of the fluid-filled cavity. Custom made MatLab programs (The Mathworks Inc., Natick, MA) were developed for processing the recorded signals following the algorithm presented in section. When this processing is applied to these signals, the overlapping of circumferential and reflections waveforms makes the identification of relevant focal spots in the backpropagation image ( $f, y$, like Fig. 2, Right) difficult, if not impossible. The cavity wall reflection contributions must be filtered out of the backpropagation images.

In this article, we propose a filtering criterion to extract the contributions of circumferential guided waves, in the backpropagation images. This criterion is: if the focal spot converges towards the central axis with increasing frequencies, then this focal spot is related to cavity wall reflections and should not be kept for the next processing step; otherwise, the focal spot is associated with circumferential guided waves and can be used for the calculation of dispersion curves. This criterion is based on the one hand, on the analysis of a simulation performed with the tool presented in section . This simulation represents the acquisition of the signals backscattered by a tube filled with glycerol but made of a fictitious highly attenuating material. The high attenuation implies that the circumferen- 
tial waves are rapidly attenuated: the backscattered wavefield is only constituted by the reflections from the tube walls. The singular value decomposition was applied to the transfer matrix of this simulation and the backpropagation images of each of the first four singular vectors are observed (Fig. 3). These images show the specific behavior of the focal spots related to reflections: they converge towards the central axis when frequency increases. This particular evolution results from the decrease of the lateral resolution with increasing frequency. Indeed, the width of the focal spot, $L$, is inversely proportional to the frequency $f: L=\frac{c_{0}}{f} \cdot \frac{F}{D}$, with $c_{0}$, the wave velocity in water, $F$ the focal length and $D$ the length of the array. The first singular vector clearly follows this tendency (top panel of Fig. 3) and the focal spots of the subsequent singular vectors align on both sides of the first central spot, seeming to converge towards the central axis (images 2, 3 and 4 of Fig. 3).

On the other hand, we know from the theory of guided waves that, on the probed frequency bandwidth and for the modes we are interested in, the phase velocity of the different modes either decreases with frequency (e.g. modes $A_{1}, S_{1}$ etc.) or is almost constant (e.g. modes $\left.A_{0}, S_{0}\right)$. As the phase velocity decreases, the distance between the pair of focal spots increases (Eq. (2)). Thus, the focal spots associated with circumferential waves diverge from the central axis or do not evolve with increasing frequency. 
Therefore, the proposed algorithm to calculate the dispersion curves is the following: (i) the interelement response matrix is measured and Fourier transformed to obtain the transfer matrix; (ii) the transfer matrix is decomposed with singular value decomposition; (iii) singular vectors are backpropagated by groups of 4 vectors to avoid a saturation of the backpropagation images with reflection contributions; (iv) on the backpropagation images, focal spots associated to circumferential guided waves are selected according to the proposed criterion, i.e. according to their evolution with frequency. The superimposition of the backpropagation image associated with the reflections on the cavity walls (obtained with simulation of the highly attenuating material) to the backpropagation images associated with simulated or experimental signals helps the operator in the selection; (v) the coordinates of the selected spots are then processed to obtain portions of dispersion curves using Eq.(2).

\section{Results \& discussion}

We proposed in this study to measure dispersion curves of circumferential waves guided by a fluid-filled bone-mimicking tube. The presence of a fluid in the cavity enables the acoustic waves to propagate and to reflect on the walls of the cavity. The contribution of these reflections on the walls of the fluid-filled cavity interfere with the contribution of circumferential guided waves we are interested in. Signals received by each array element after excitation with a plane wave of an empty 
tube, of a fluid-filled tube and of a fluid-filled tube of highly attenuating material were simulated (Fig. 4). Due to the multiple reflection echoes, the circumferential wave contributions can hardly be seen in the case of the fluid-filled tube. The last image of the figure 4 indicates that, in the case of the fluid-filled highly attenuating tube, the only contribution to the backscattered wavefield actually comes from the reflections on the cavity walls.

The backpropagation images obtained with the first four singular vectors of the transfer matrix of the fluid-filled tube measured and simulated depict the superposition of the cavity reflection and the circumferential wave contributions (Fig. 5). The characteristic pattern of the reflections (observed with the simulation of the highly attenuating tube, Fig. 3) can be recognized, modified in some areas by the contributions of the circumferential guided waves. The comparison of the singular vector amplitudes of the fluid-filled tube and of the fluid-filled highly attenuating tube, at one frequency (Fig. 6) illustrates the fact that the singular value decomposition does not enable to separate the cavity reflection and the circumferential waves contributions. In the simulation of a fluid-filled tube of a highly damping material the singular vector amplitudes corresponds to typical patterns (intermediate case between Gaussian and Legendre polynomials) associated with specular reflections. ${ }^{23,27}$ In the simulation of the fluid-filled 
bone-mimicking tube, the singular vectors present the same type of motif but distorted by the contribution of circumferential waves: each singular vector conveys information about both circumferential waves and cavity reflections.

The criterion proposed in the former section is applied: if a focal spot does not converge towards the central axis with increasing frequency, then this focal spot is associated with circumferential guided waves. This criterion is applied to the backpropagation images obtained with the singular vectors 2 to 5 of the simulated and measured transfer matrix (Fig. 7 and 8): the focal spots kept for the next step of the processing are indicated by a solid line while the focal spots assumed to correspond to cavity reflection contributions are represented by a dashed line. The backpropagation images of the singular vectors 6 to 9 and 10 to 13 were also processed to obtain the location of circumferential wave focal spots.

The distances between chosen focal spots were converted into dispersion curves using Eq.(2). Portions of circumferential wave dispersion curves obtained from experimentally recorded signals are compared with those obtained from simulated signals (Fig. 9). Most of the simulated portions are experimentally observed, indicating the quality of our experimental set-up. However, some portions are obtained only in the experiment or only in the simulation. This discrepancy can be explained by differences of signal amplitudes: the singular value decom- 
position is slightly different between experiment and simulation. Hence, the backpropagation image of the highest singular vectors obtained from simulation is not strictly identical to the corresponding image calculated from experiment (Fig. 7 and 8). Specifically, the focal spots of the backpropagation image of the experiment, for some frequencies, are not collocated with those obtained from simulation. Thus, in some areas of the backpropagation images, the focal spots from experiment (respectively simulation) are eliminated because they are superimposed with reflection focal spots whereas focal spots from simulation (respectively experiment), being slightly different are not superimposed and not eliminated. However, these portions obtained only in simulation or experiment seem nevertheless to be reliable: they are aligned with points or portions obtained in both experiment and simulation. The comparison between experimental points and theoretical dispersion curves of a doubly fluid-loaded plate enables to identify the observed portions with modes $A, S, A_{0}, S_{0}, A_{1}, S_{1}$ and $A_{3}$ (Fig. 9). Points near the cut-off frequency of $S_{2}$ are also observed. We can notice that, compared to our previous results on an empty tube, ${ }^{13}$ the portions of the modes of the fluid-filled tube are experimentally observed on smaller bandwidths. This is a consequence of the proposed filtering method. Actually, this criterion only enables distinguishing the contributions of the circumferential waves when the corresponding focal spots are distinct from the reflection focal spots. If the focal spots are collocated, the reflection contributions being of a larger amplitude, these spots will not be taken into account to calculate the dispersion 
curves. Attenuation may also play a role. When the tube is filled with a fluid, attenuation of the circumferential guided waves by leakage in both the external medium and the cavity increases. We have checked using Disperse that areas of the $(f, k)$ plane where no dispersion branch is obtained actually correspond to high values of attenuation (Fig. 10).

The selection and matching of the focal spots in the backpropagation images (Fig. 7, 8) were performed manually for this study. However, these operations could be automated. Indeed, the tasks involved in these operations are identification, matching of alignment of focal spots and then comparison of two sets of aligned focal spots obtained from the two backpropagation images.

Differences between the cortical bone-mimicking phantom filled with glycerol considered in this study and a human femoral neck should be acknowledged. First, the acoustical impedance of glycerol being higher than that of marrow, the acoustical impedance mismatch is smaller than the actual mismatch between cortical bone and marrow. Thus, for the femur neck, it is expected that less energy would be transmitted to the medullary cavity and that the circumferential waves would be less attenuated by radiation leakage in the cavity. Second, the presence of trabecular bone on the endosteal cortical side is not considered in the study. Because trabecular bone strongly attenuates (by absorption and scattering) the waves that are transmitted in the cavity, the reflections should be a less critical issue in real life. Finally, the soft tissues surrounding the neck were not taken into account. The 
soft tissues, unlike the medium used in our study (water), are heterogeneous. The influence of this heterogeneity on the applicability of the method must be studied before considering in vivo application.

A limitation of the study is that the femoral neck geometry was approximated by a tube of circular cross-section. The method proposed in this article will serve as a basis to undertake studies with more realistic geometries.

\section{Conclusion}

An adaptation of the DORT method was proposed to measure the wavenumbers of circumferential guided waves in a fluid-filled cylindrical cortical bone-mimicking phantom. Eight guided modes of the bone-mimicking phantom obtained from experimental and simulated data were identified and their dispersion curves were in good agreement with theory. This study represents a step towards the ultrasonic characterization of cortical shell of the femoral neck. However, it is of more general interest. It may for instance find applications for the ultrasonic inspection of immersed composite pipes.

\section{REFERENCES}

1. O. Strom, F. Borgstrom, J. A. Kanis, J. Compston, C. Cooper, E. McCloskey, and B. Jonsson, "Osteoporosis: burden, health care provision and opportunities in the EU", Archives of Osteoporosis 6, 59-155 (2011). 
2. S. Schuit, M. van der Klift, A. Weel, C. de Laet, H. Burger, E. Seeman, A. Hofman, A. Uitterlinden, J. van Leeuwen, and H. Pols, "Fracture incidence and association with bone mineral density in elderly men and women: the Rotterdam Study", Bone 34, 195 $-202(2004)$.

3. T. M. Keaveny, D. L. Kopperdahl, L. J. Melton, P. F. Hoffmann, S. Amin, B. L. Riggs, and S. Khosla, "Age-dependence of femoral strength in white women and men", J. Bone Miner. Res. 25, 994-1001 (2010).

4. P. Laugier, "Instrumentation for in vivo ultrasonic characterization of bone strength", IEEE Trans. Ultrason. Ferroelectr. Freq. Control 55, 1179-96 (2008).

5. D. Marshall, O. Johnell, and H. Wedel, "Meta-analysis of how well measures of bone mineral density predict occurence of osteoporotic fractures", BMJ 312, 1254-1259 (1996).

6. R. Barkmann, S. Dencks, P. Laugier, F. Padilla, K. Brixen, J. Ryg, A. Seekamp, L. Mahlke, A. Bremer, M. Heller, and C. C. Gluer, "Femur ultrasound (FemUS)first clinical results on hip fracture discrimination and estimation of femoral BMD", Osteoporos. Int. 21, 969-76 (2009).

7. G. Holzer, G. von Skrbensky, L. A. Holzer, and W. Pichl, "Hip fractures and the 
contribution of cortical versus trabecular bone to femoral neck strength", J. Bone Miner. Res. 24, 468-474 (2009).

8. R. M. Zebaze, A. Ghasem-Zadeh, A. Bohte, S. Iuliano-Burns, M. Mirams, R. I. Price, E. J. Mackie, and E. Seeman, "Intracortical remodelling and porosity in the distal radius and post-mortem femurs of women: a cross-sectional study", Lancet $\mathbf{3 7 5}, 1729$ $36(2010)$.

9. J.-G. Minonzio, J. Foiret, M. Talmant, and P. Laugier, "Impact of attenuation on guided mode wavenumber measurement in axial transmission on bone mimicking plates", J. Acoust. Soc. Am. 130, 3574-3582 (2011).

10. J. Grondin, Q. Grimal, K. Engelke, and P. Laugier, "Potential of first arriving signal to assess cortical bone geometry at the hip with QUS: a model based study", Ultrasound Med. Biol. 36, 656-66 (2010).

11. Q. Grimal, J. Grondin, S. Guérard, R. Barkmann, K. Engelke, C.-C. Glüer, and P. Laugier, "Quantitative ultrasound of cortical bone in the femoral neck predicts femur strength: results of a pilot study", J. Bone Miner. Res. (2012).

12. D. Ta, W. Wang, Y. Wang, L. H. Le, and Y. Zhou, "Measurement of the dispersion and attenuation of cylindrical ultrasonic guided waves in long bone", Ultrasound Med. Biol. 35, 641-52 (2009). 
13. P. Nauleau, E. Cochard, J. G. Minonzio, Q. Grimal, P. Laugier, and C. Prada, "Characterization of circumferential guided waves in a cylindrical cortical bone-mimicking phantom", J. Acoust. Soc. Am. 131, EL289-EL294 (2012).

14. C. Prada, S. Manneville, D. Spoliansky, and M. Fink, "Decomposition of the time reversal operator: Detection and selective focusing on two scatterers", J. Acoust. Soc. Am. 99, 2067-2076 (1996).

15. C. Prada and M. Fink, "Separation of interfering acoustic scattered signals using the invariants of the time-reversal operator. application to lamb waves characterization", J. Acoust. Soc. Am. 104, 801-807 (1998).

16. J. L. Thomas, P. Roux, and M. Fink, "Inverse scattering analysis with an acoustic time-reversal mirror", Phys. Rev. Lett. 72, 637-640 (1994).

17. K. L. Williams and P. L. Marston, "Backscattering from an elastic sphere: SommerfeldWatson transformation and experimental confirmation", J. Acoust. Soc. Am. 78, 1093 $-1102(1985)$.

18. M. Granke, Q. Grimal, A. Saïed, P. Nauleau, F. Peyrin, and P. Laugier, "Change in porosity is the major determinant of the variation of cortical bone elasticity at the millimeter scale in aged women", Bone 49, 1020-1026 (2011). 
19. S. Goss, R. Johnston, and F. Dunn, "Compilation of empirical ultrasonic properties of mammalian tissues. II", J. Acoust. Soc. Am. 68, 93-108 (1980).

20. H. Blain, P. Chavassieux, N. Portero-Muzy, F. Bonnel, F. Canovas, M. Chammas, P. Maury, and P. D. Delmas, "Cortical and trabecular bone distribution in the femoral neck in osteoporosis and osteoarthritis", Bone 43, 862-8 (2008).

21. A. S. Aula, J. Toyras, M. A. Hakulinen, and J. S. Jurvelin, "Effect of bone marrow on acoustic properties of trabecular bone - 3D finite difference modeling study", Ultrasound Med. Biol. 35, 308-18 (2009).

22. G. V. Frisk and H. Überall, "Creeping waves and lateral waves in acoustic scattering by large elastic cylinders", J. Acoust. Soc. Am. 59, 46-54 (1976).

23. A. Aubry, J. de Rosny, J.-G. Minonzio, C. Prada, and M. Fink, "Gaussian beams and Legendre polynomials as invariants of the time reversal operator for a large rigid cylinder", J. Acoust. Soc. Am. 120, 2746-2754 (2006).

24. J.G. Minonzio, F. Philippe, C. Prada, and M. Fink, "Characterization of an elastic cylinder and an elastic sphere with the time-reversal operator : application to the sub-resolution limit", Inverse Probl. 24, (2008).

25. B. N. Pavlakovic, M. J. S. Lowe, D. N. Alleyne, and P. Cawley, Review of progress in 
quantitative NDE, volume 16, chapter Disperse: a general purpose program for creating dispersion curves, 185-192, edited by D. Thompson and D. Chimenti, (Plenum, New York) (1997).

26. M. F. M. Osborne and S. D. Hart, "Transmission, reflection, and guiding of an exponential pulse by a steel plate in water. I. Theory", J. Acoust. Soc. Am. 17, 1-18 (1945).

27. S. Komilikis, C. Prada, and M. Fink, "Characterization of extended objects with the DORT method", volume 104, 1401-1404 (1996). 


\section{Figure Captions}

Figure 1. A tube of diameter $D$ immersed in water is excited with an array of transducers. A circumferential wave of velocity $c_{\varphi}$ is generated in $A$. This wave propagates in the shell and radiates into the external medium. The radiated wave recorded by the array appears to come from a virtual source $B^{\prime}$, located on the median axis $\Delta$ of the tube. Reciprocally, a circumferential wave is generated in $B$, and the radiated wave is seen coming from $A^{\prime}$ for the array.

Figure 2. (Top) Backpropagation images $x, y$ at 0.6 and $1 \mathrm{MHz}$. Several virtual sources can be identified $\left(A_{1}^{\prime}, A_{2}^{\prime}, A_{3}^{\prime}\right.$ and $\left.B_{1}^{\prime}, B_{2}^{\prime}, B_{3}^{\prime}\right)$. (Bottom) Backpropagation image observed on the line $\Delta(x=0)$, at several frequencies. The distance between the virtual sources $A_{i}^{\prime}$ and $B_{i}^{\prime}$ is measured on this image and converted into phase velocity using Eq.(2).

Figure 3. Backpropagation images of the first four singular vectors of the simulated transfer matrix of a phantom filled with glycerol made of a fictitious highly attenuating material. A specific behavior of focal spots related with reflections can be noticed: they converge towards the central axis as the frequency increases.

Figure 4. Simulated signals scattered by three phantoms : an empty phantom (a) previously studied $^{13}$, the fluid-filled phantom studied here (b) and a highly attenuating phantom filled with the same fluid (c). Each line of these images represents, with a color map, the signals received by one of the 128 transducers after a plane wave emission. Two kinds of contributions 
can be distinguished : radiated circumferential waves [(a) and (b)] and multiple reflections on the cavity walls, labeled R. [(b) and (c)]. By comparison of the three images, we can notice that the radiated circumferential waves overlap with the multiply reflected waves. Bold black lines delineate the part of signals containing information on circumferential waves used for the processing.

Figure 5. Backpropagation images of the first four singular vectors of experimental (Left) and simulated (Right) transfer matrices of a fluid-filled bone-mimicking tube.

Figure 6. The first four singular vectors $(\mathrm{SV})$ at the central frequency $(1 \mathrm{MHz})$ corresponding to the simulated transfer matrix of the studied phantom, a cortical bone-mimicking tube filled with glycerol (solid line) and a phantom filled with glycerol made of a fictitious highly attenuating material (dashed line). The typical pattern of reflections ${ }^{23,27}$ can be observed for the second phantom.

Figure 7. Backpropagation image of the singular vectors 2 to 5 of the simulated transfer matrix of the studied bone-mimicking phantom filled with glycerol. On this image, series of focal spots associated with circumferential waves which do not converge towards the central axis (solid line) are selected. The other focal spots corresponding to reflections are excluded(dashed line).

Figure 8. Backpropagation image of the singular vectors 2 to 5 of the measured transfer matrix of the studied phantom. Similarly to Fig. 7, the solid line corresponds to focal 
spots identified as circumferential wave contributions by the operator and the dashed line corresponds to focal spots identified as reflection contributions.

Figure 9. Identification of dispersion curves portions obtained, in the fluid-filled case [ Fig. 4(b) ], from experimentally recorded signals (o) and simulated signals $(+)$ with theoretical predictions from a doubly fluid-loaded plate $(\bullet)$. Portions of $A, S, A_{0}, S_{0}, A_{1}, S_{1}$ and $A_{3}$ as well as cut-off frequency of $S_{2}$ are measured. Areas where no points are obtained have been found to correspond to high values of attenuation (Fig.10).

Figure 10. Attenuation due to radiation in the external media calculated by Disperse for a plate loaded with water one side and glycerol on the other side. High values of attenuation in some areas explain why no portions of dispersion curves are observed. 


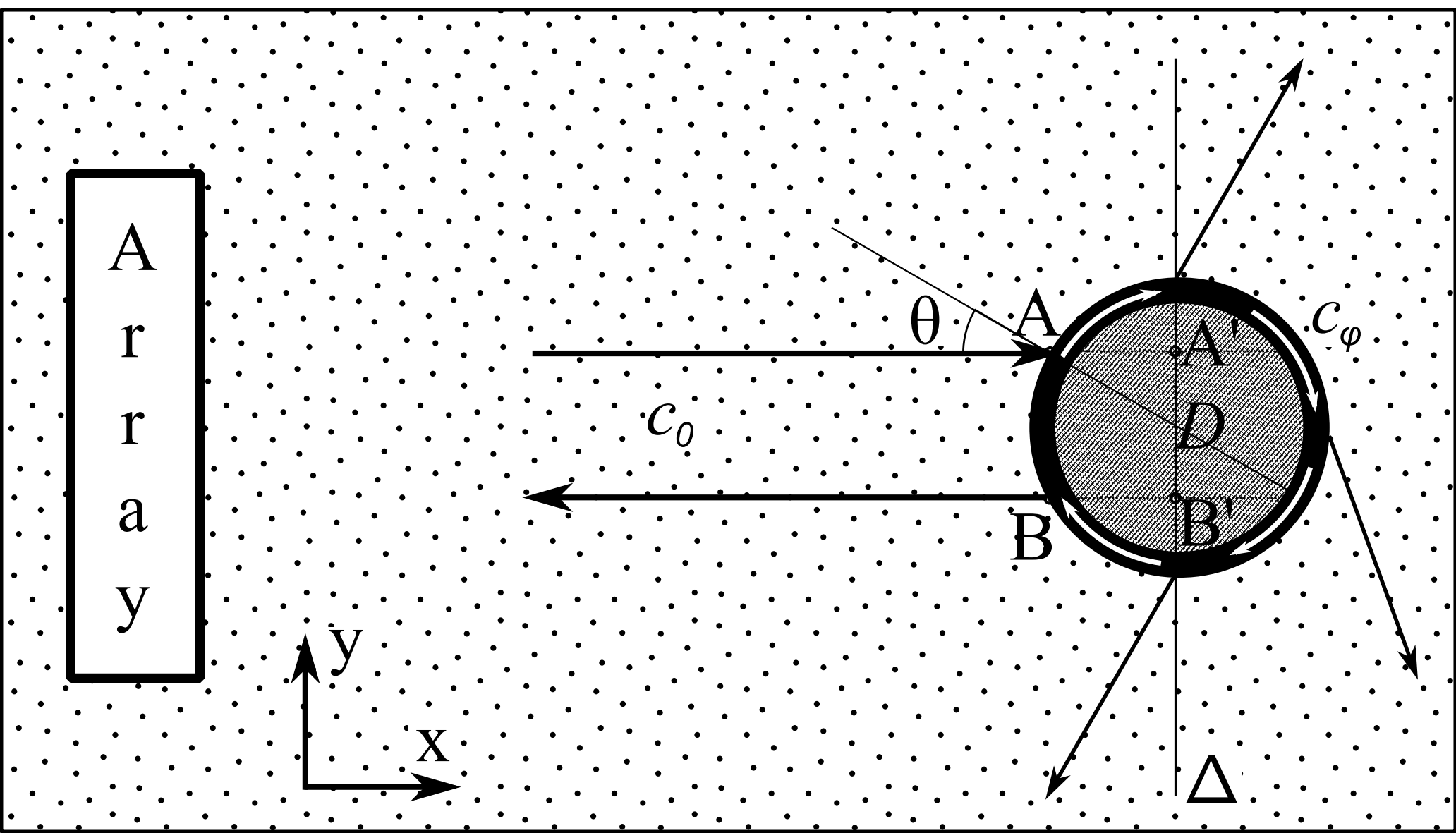



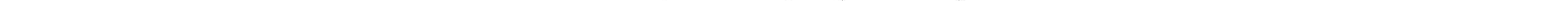
18

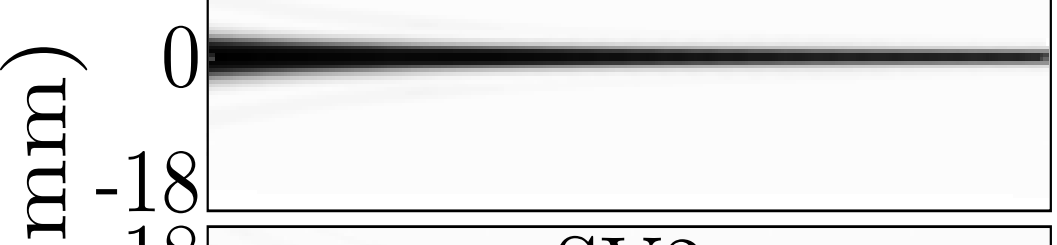

18

0

U

$\longrightarrow$

बु -18

。

\& 0

.

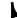

$x$

ชి

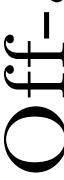

$-18$

18

0

0
-18
SV1

SV2

SV3

0.4

0.6

0.3

0.5

0.3

0.1

SV4

0.4

0.2 
a- Empty tube

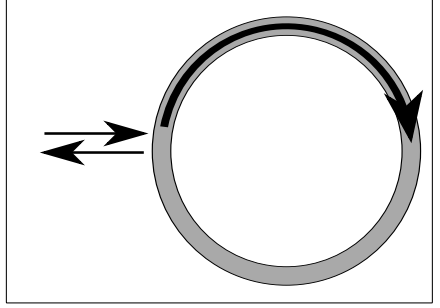

b- Fluid filled tube

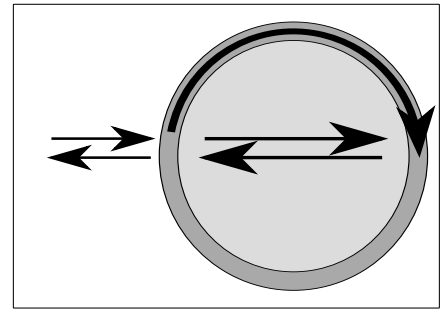

c- Fluid filled highly attenuating tube

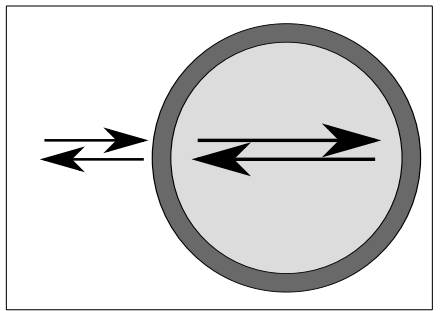

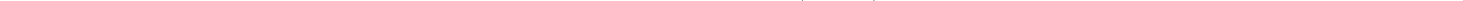




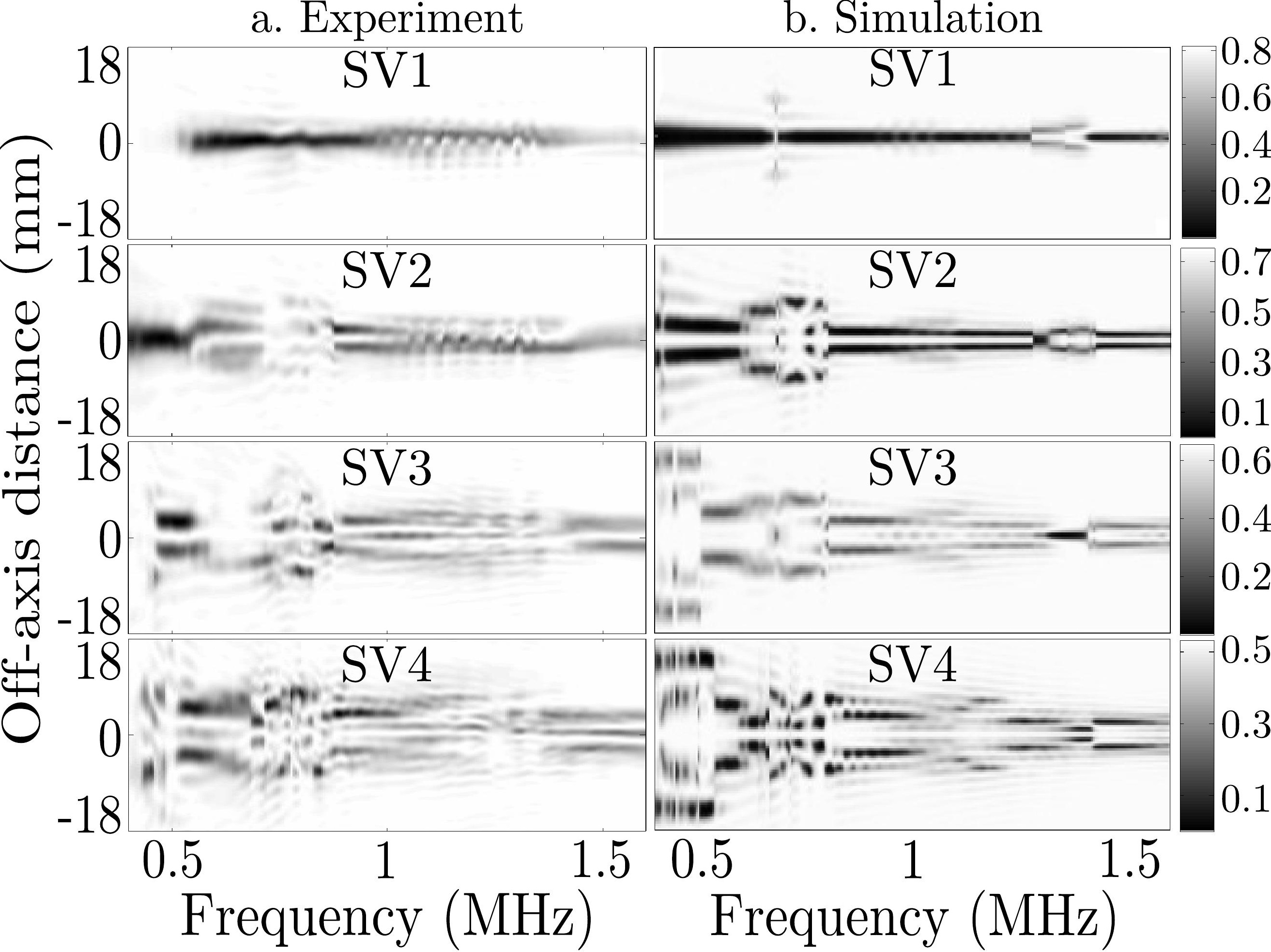


— Fluid-filled tube

..... Fluid-filled highly attenuating tube

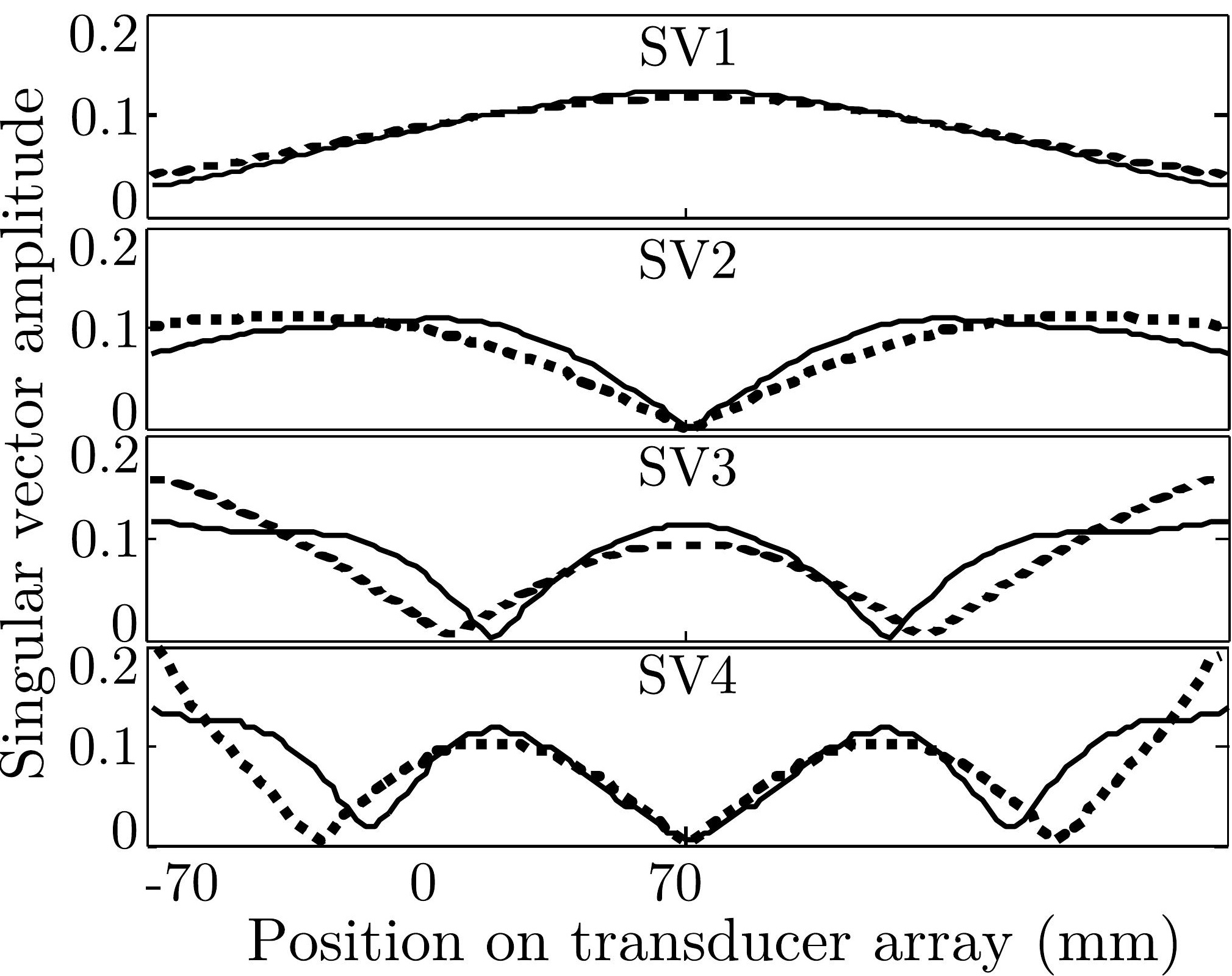




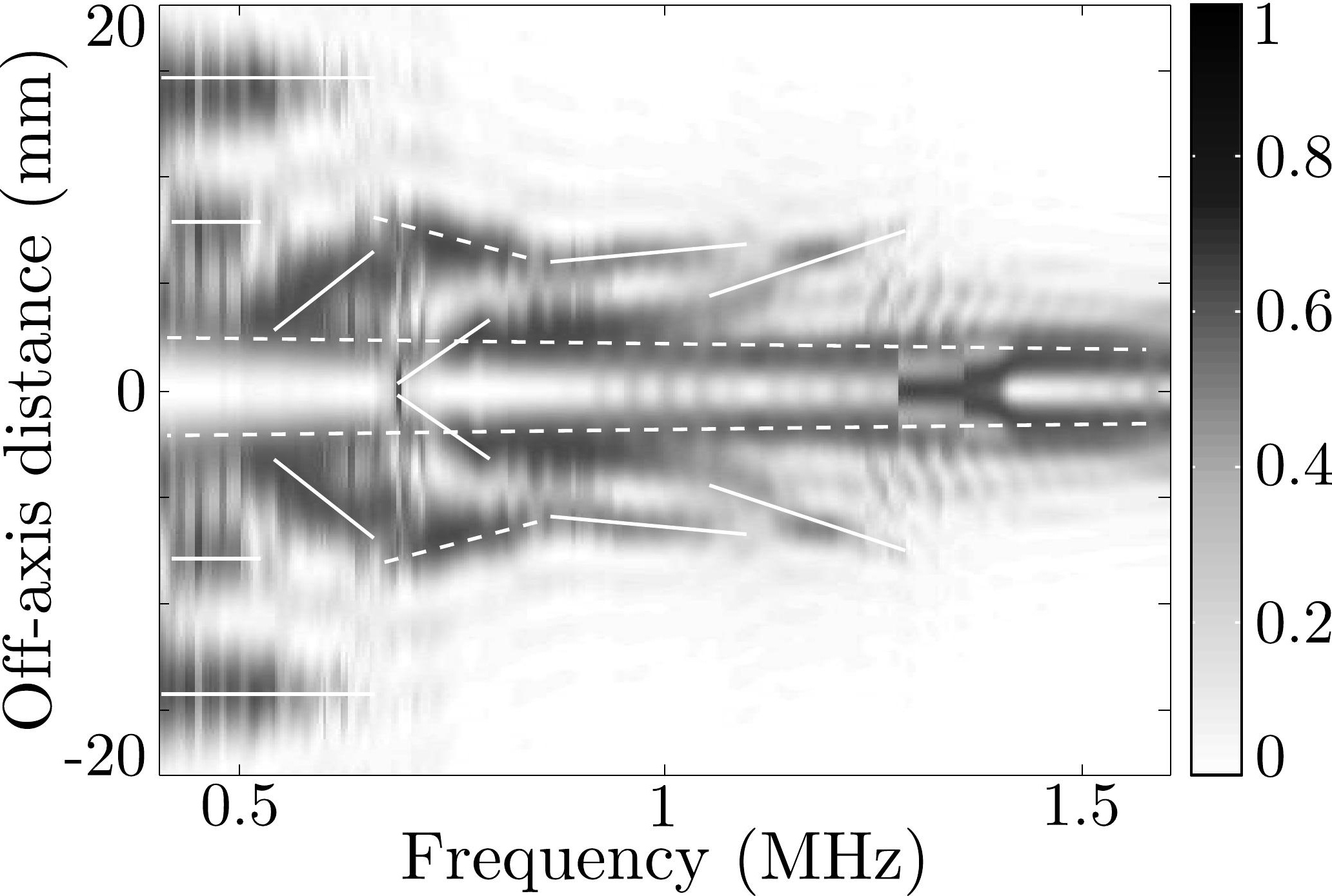




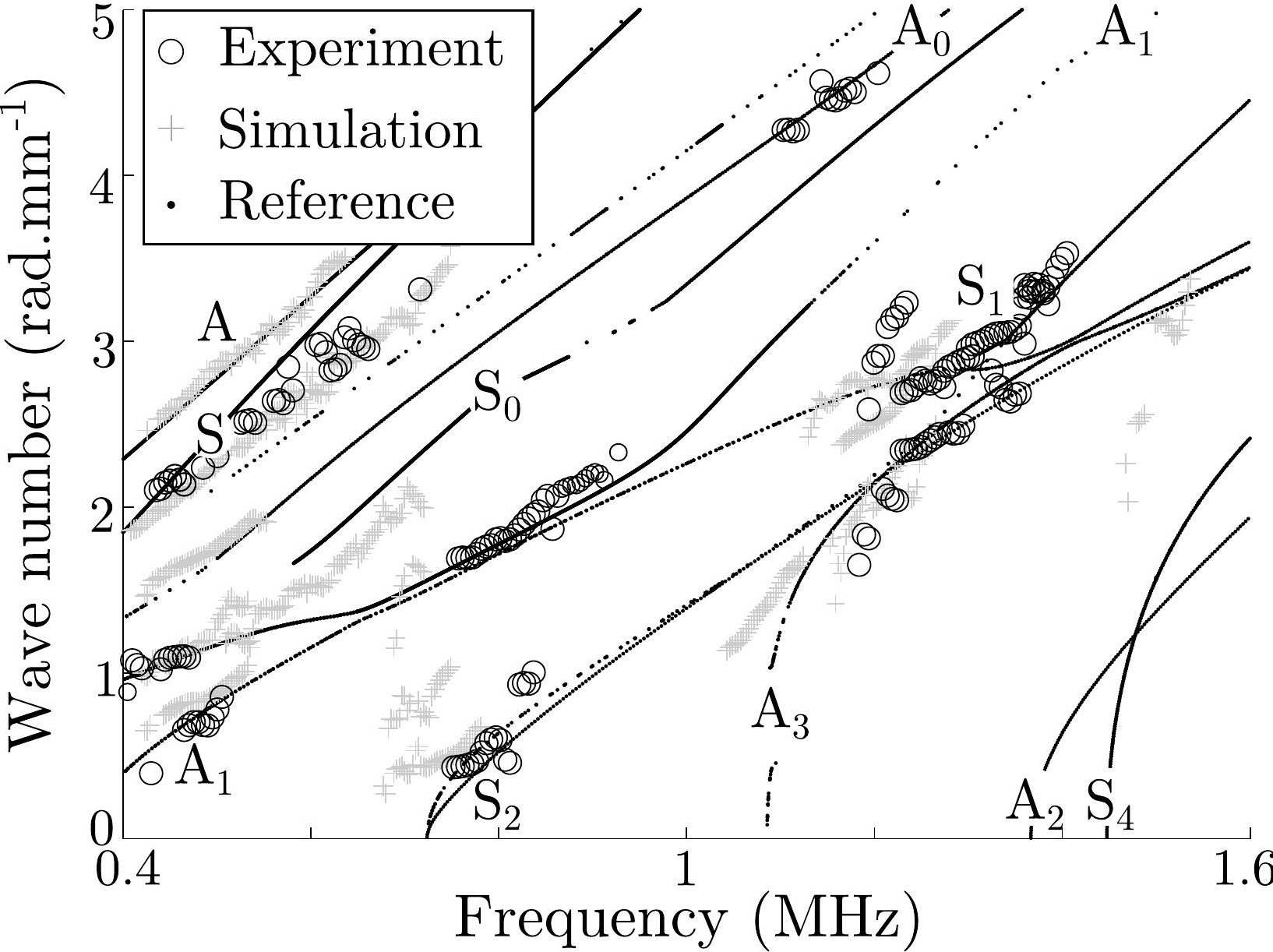




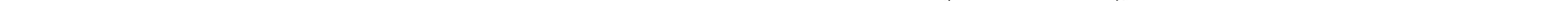

